\title{
Borderline Personality and Alexithymia among Female College Students with Interpersonal Problems
}

\section{Mehrangiz Peyvastegar*}

Faculty of Educational Sciences and Psychology, Alzahra University, Tehran, Iran

\section{Article Info:}

\section{A BSTRACT}

Introduction: Studies have shown that borderline personality and alexithymia could affect the course of interpersonal problems. The aim of this study was to evaluate borderline personality characteristics and alexithymia among female college students with interpersonal problems. Materials and Methods: Research population included 150 female students in AL Zahra University who were received above cutoff scores in the inventory of interpersonal problems. The instruments used in this study were the inventory of interpersonal problems, the Toronto Alexithymia Scale (TAS-20), and the Personality Assessment Inventory Borderline Scale (PAI-BOR). Results: The results showed that difficulty identifying feelings and impulsive behaviors were predictors of interpersonal problems. Conclusion: These findings indicate the role of borderline personality and alexithymia in interpersonal problems. These results can be useful in intervention programs in treatments of interpersonal problems.

*Corresponding Author: Mehrangiz Peyvastegar

E-mail: mpaivastegar@alzahra.ac.ir
Key words:

1. Behavior

2. Therapeutics

3. Female 


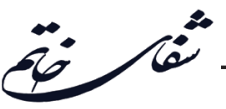

اختلال شخصيت مرزى و ناگويى هيجانى در ميان دختران دانشجوى داراى مشكلات بين فردى

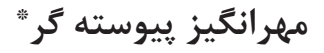

دانشكده علوم تربيتى و روانشناسى، دانشخاه الزهرا، تهران، ايران

\section{إطلاعات مقاله:}

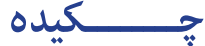

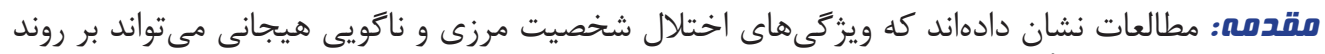

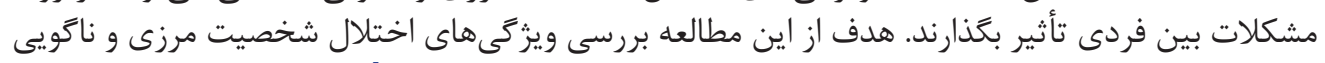

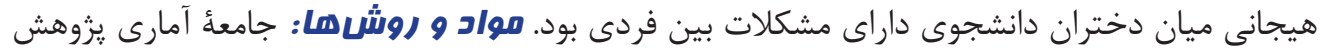

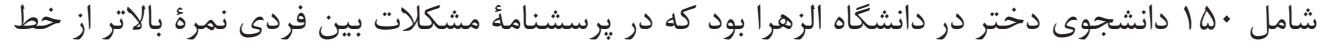

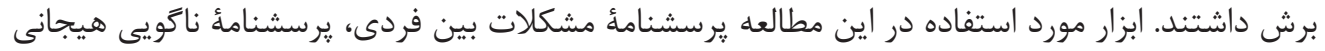

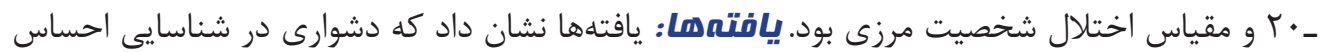

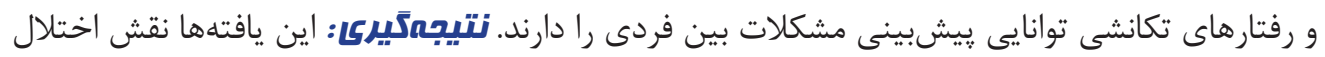

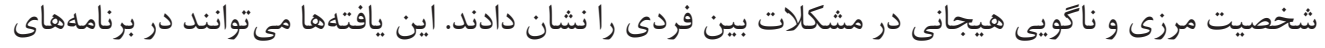

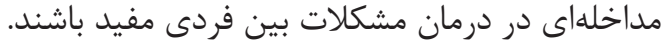

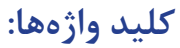

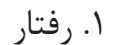

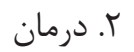

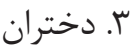

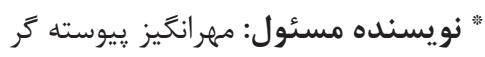
آدرس الكترونيكى: mpaivastegar@alzahra.ac.ir 


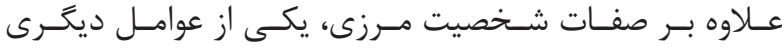

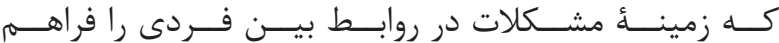

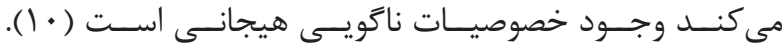

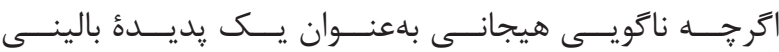

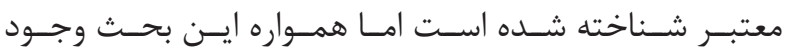

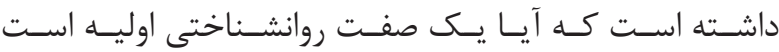

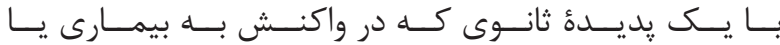

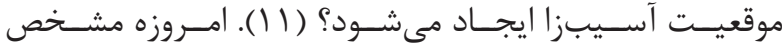

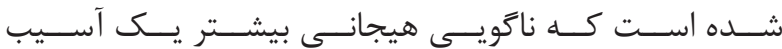

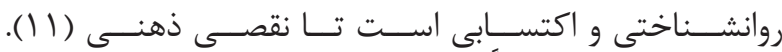

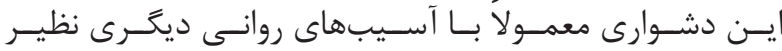

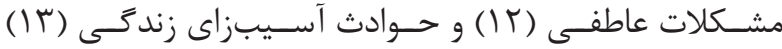

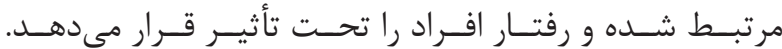

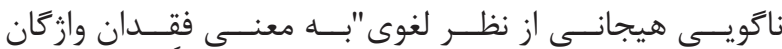

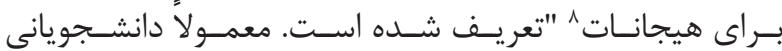

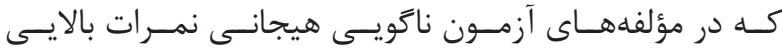

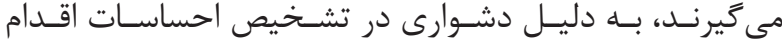

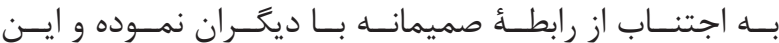

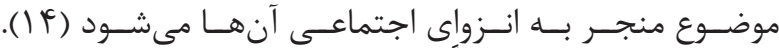

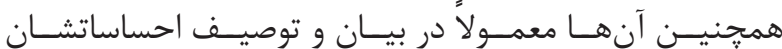

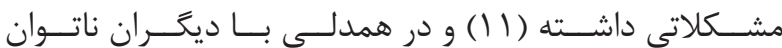

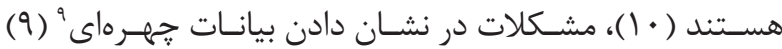

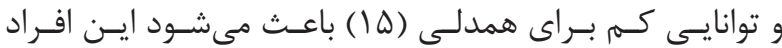

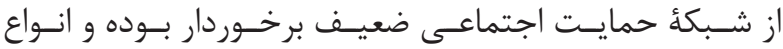

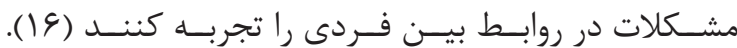

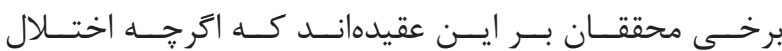

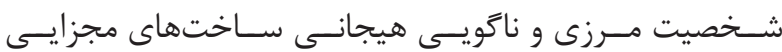

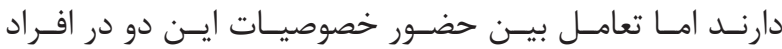

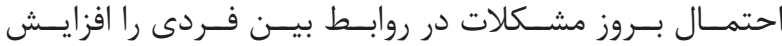

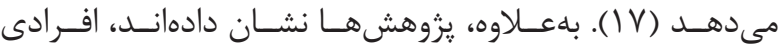

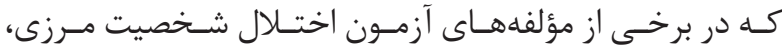

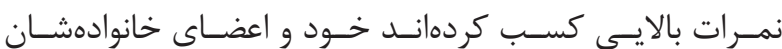

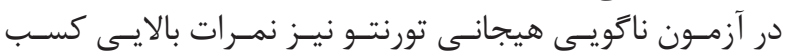

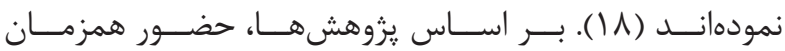

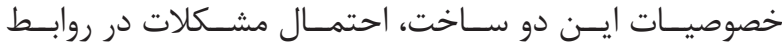

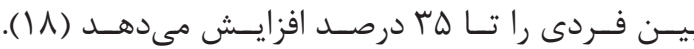

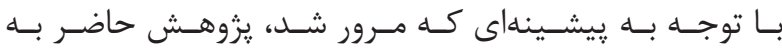

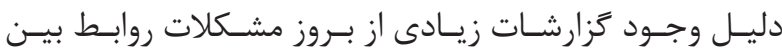

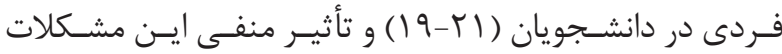

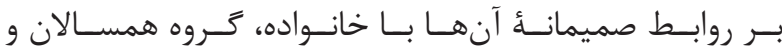

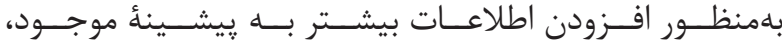

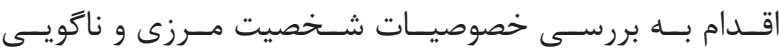

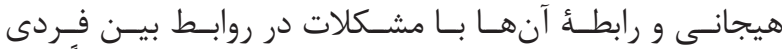

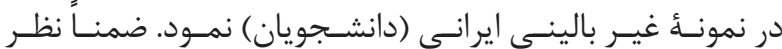

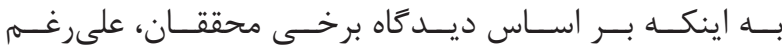

${ }^{1}$ Problems in interpersonal relationships

${ }^{2}$ Linehan

${ }^{3}$ De Rick and Vanheule

${ }^{4}$ McMurran, Huband and Overton

${ }^{5}$ Diagnostic and statistical manual of mental disorders

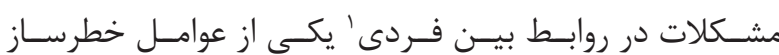

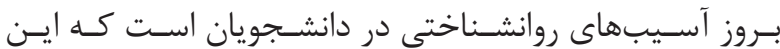

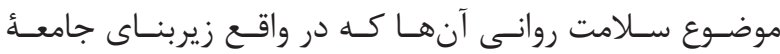

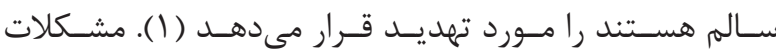

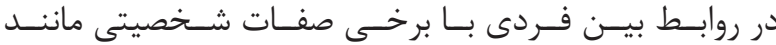

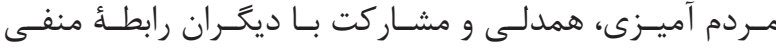

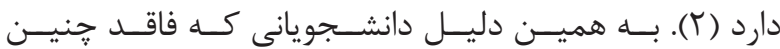

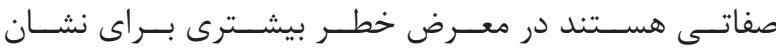

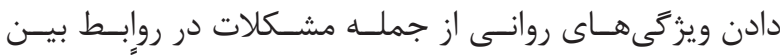

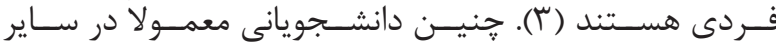

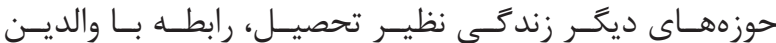

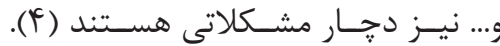

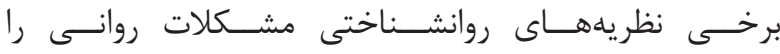

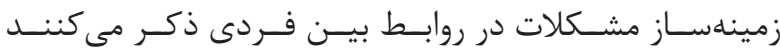

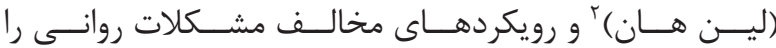

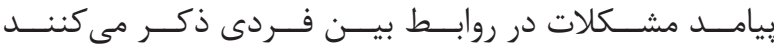

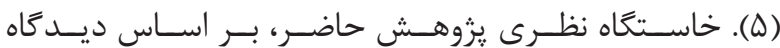

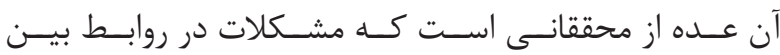

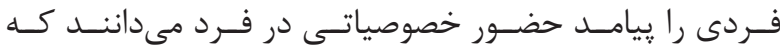

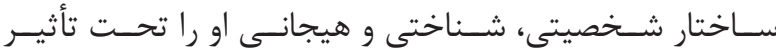

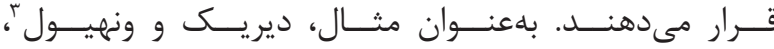

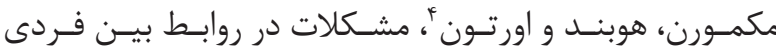

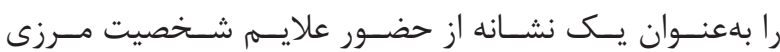

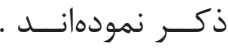

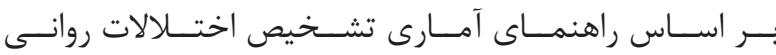

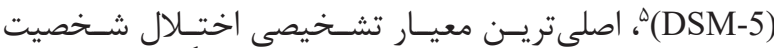

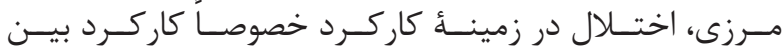

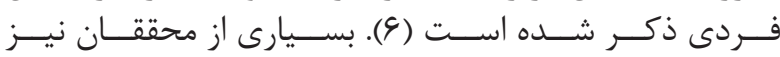

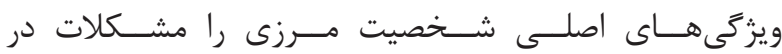

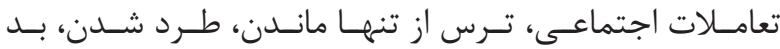

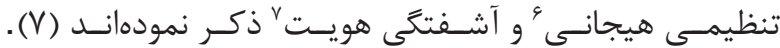

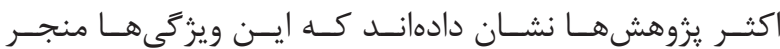

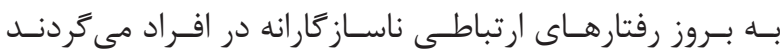

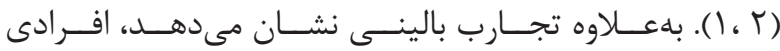

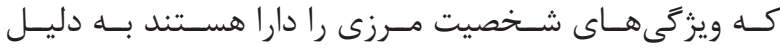

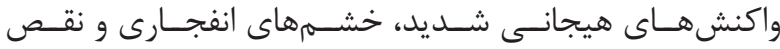

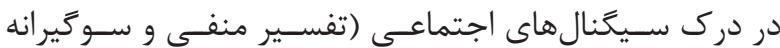

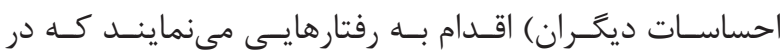

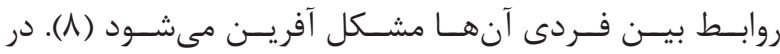

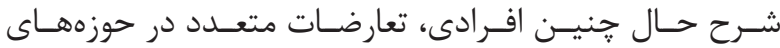

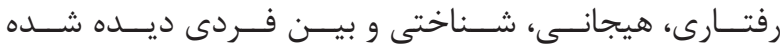

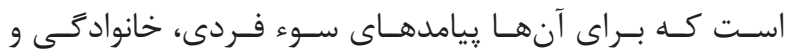

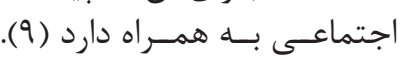

\footnotetext{
${ }^{6}$ Affective dysregulation

${ }^{7}$ Identity disturbances

${ }^{8}$ Without words for emotions

${ }^{9}$ Facial expressions
} 


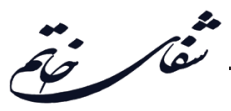

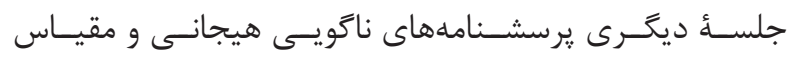

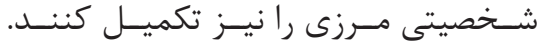

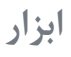

جهــت گـردآورى اطلاعـات در يزوهــش حاضــر از ســه ابـزار

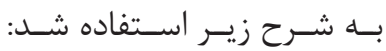

يرسشنامة مشكلات بين فردى

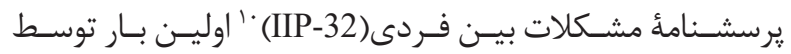

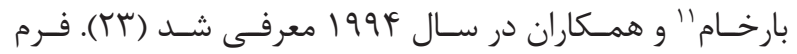

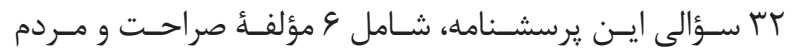

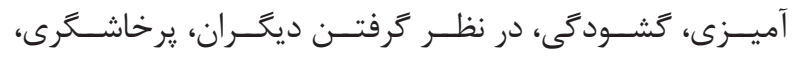

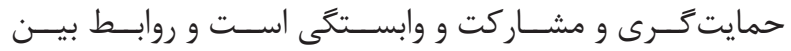

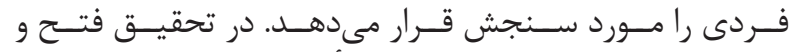

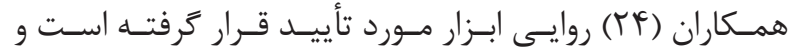

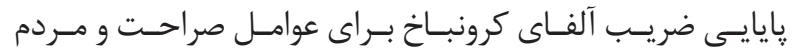

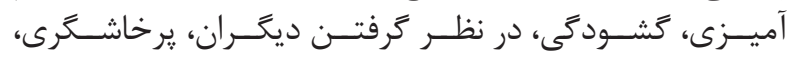

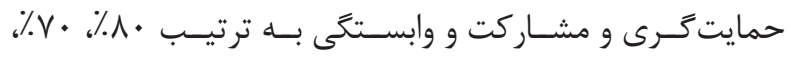

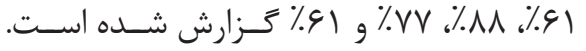

مقياس شخصيت مرزى

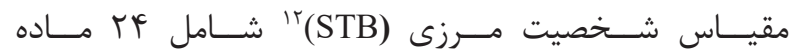

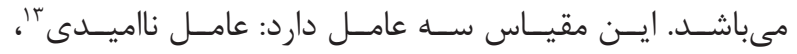

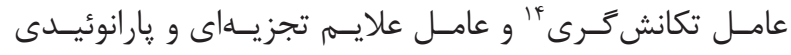

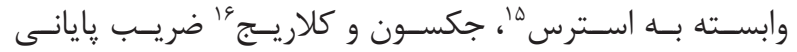

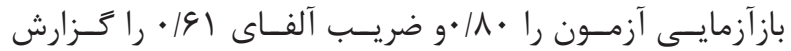

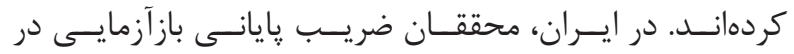

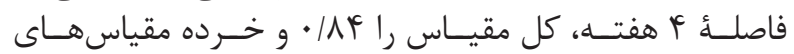

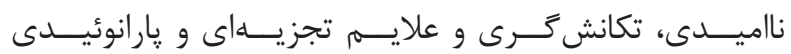

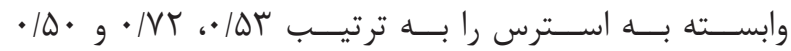

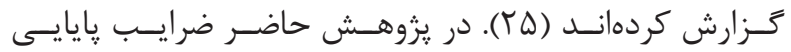

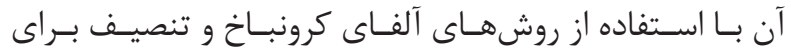

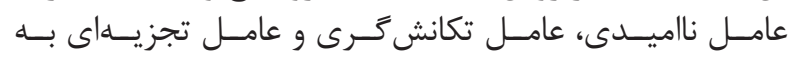

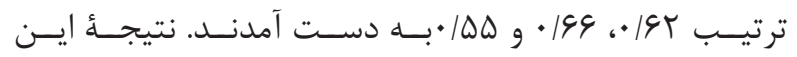

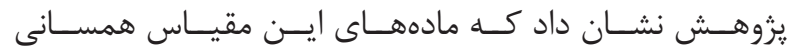

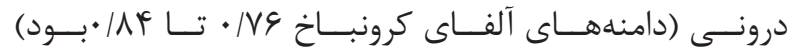
خوبسى دارد.

\section{r·- برسشنامة ناگويى هيجانى}

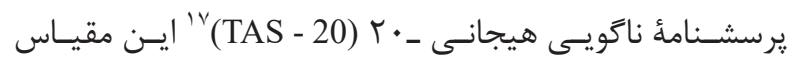

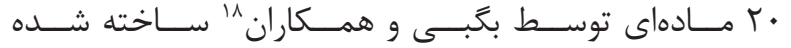

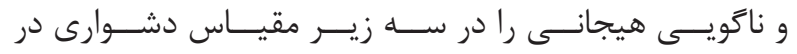

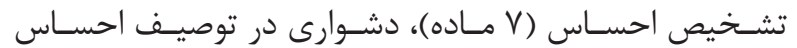

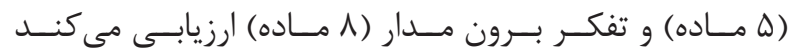

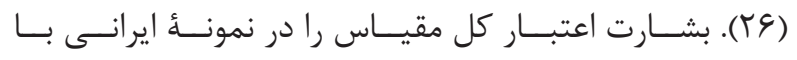

${ }^{10}$ Inventory of interpersonal problems-32

${ }^{11}$ Barkham

${ }^{12}$ Borderline personality scale

${ }^{13}$ Hopelessness

${ }^{14}$ Impulsivity

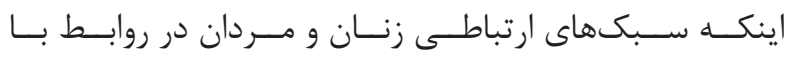

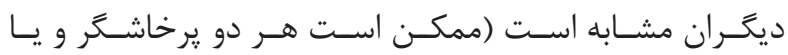

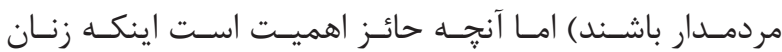

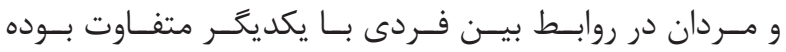

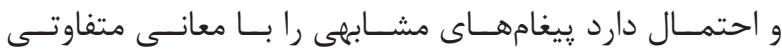

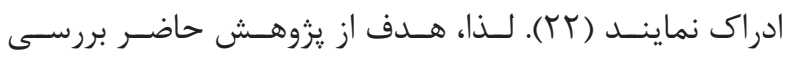

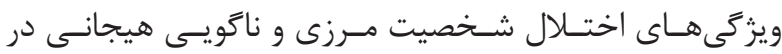

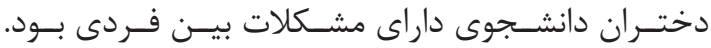

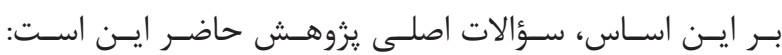

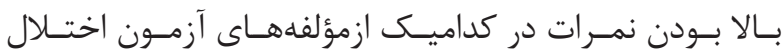

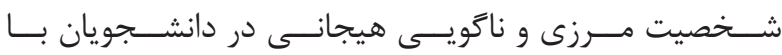

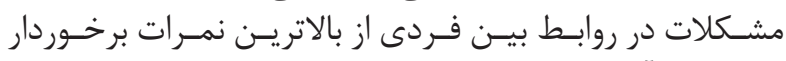

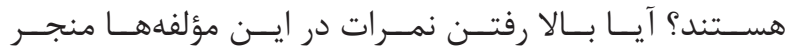

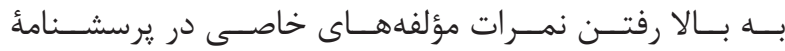

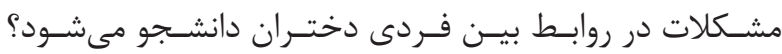

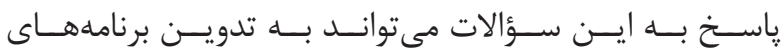

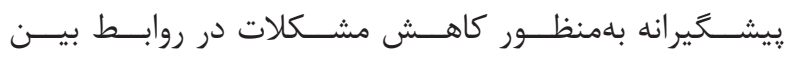

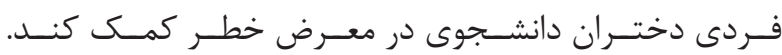

\section{مواد و روشها} جامعلة آمارى و روش نمونه كيرى

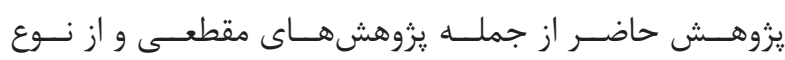

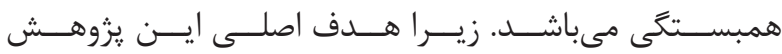

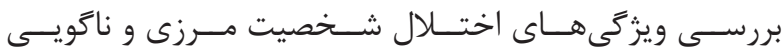

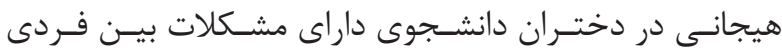

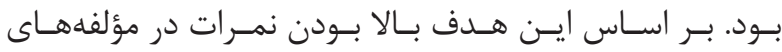

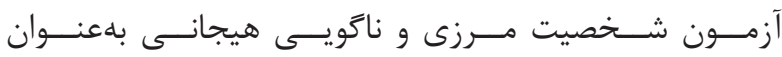

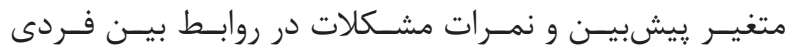

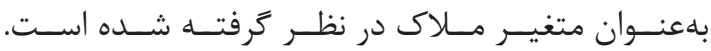

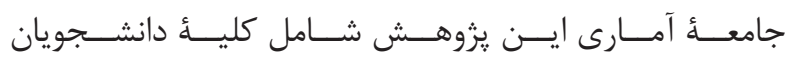

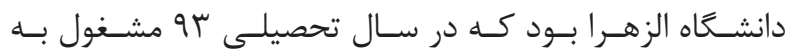

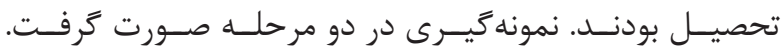

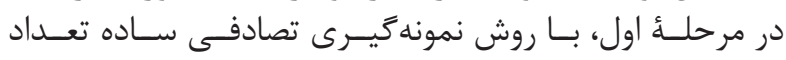

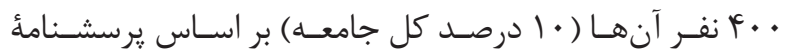

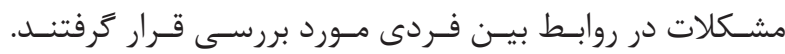

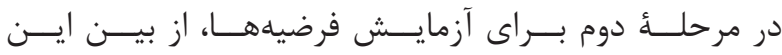

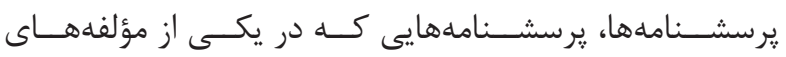

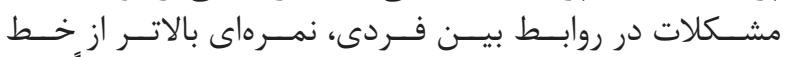

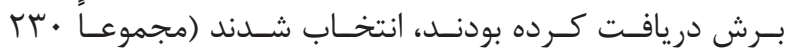

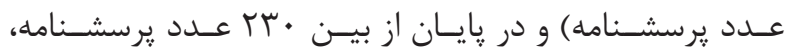

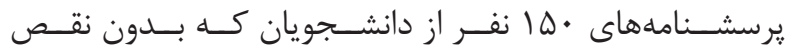

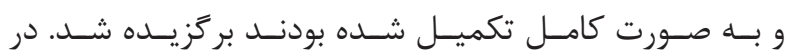

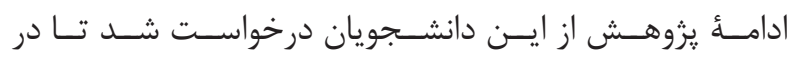

${ }^{15}$ Stress related paranoid/ dissociative symptoms

${ }^{16}$ Jackson and Claridge

${ }^{17}$ Toronto alexithymia scale

${ }^{18}$ Baghby 


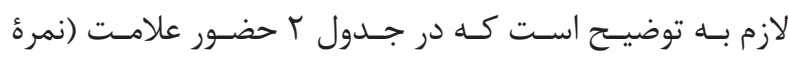

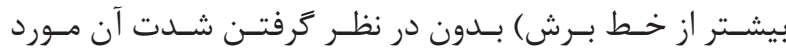

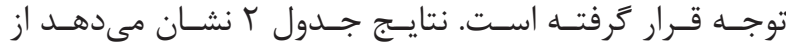

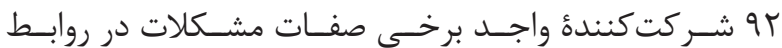

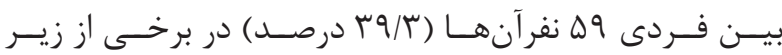

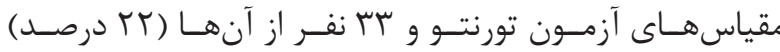

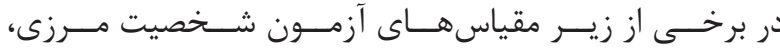

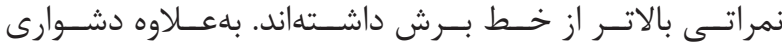

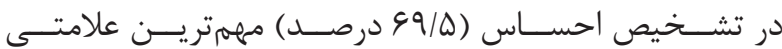

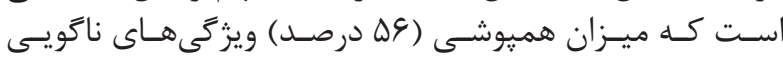

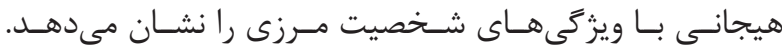

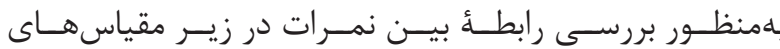

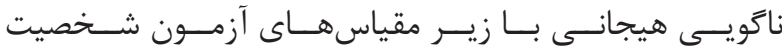

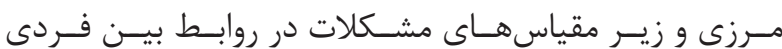

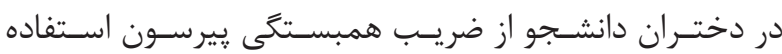

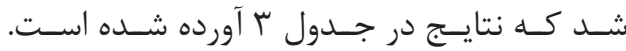

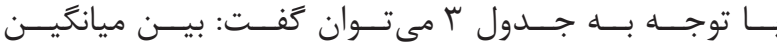

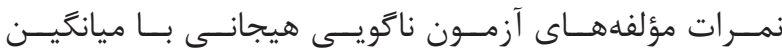

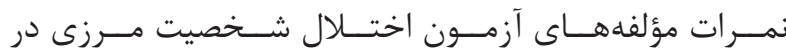

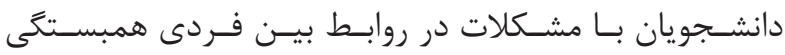

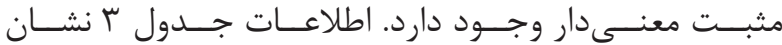

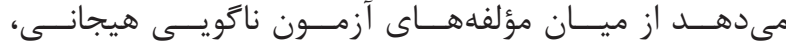

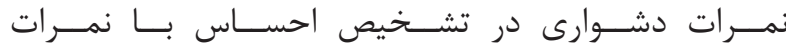

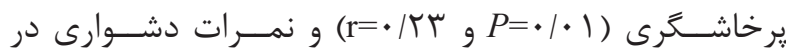

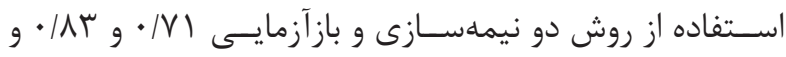

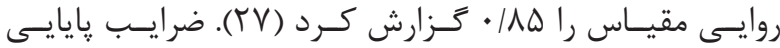

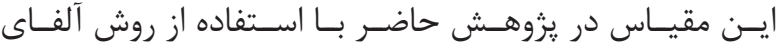

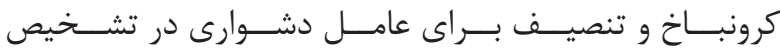

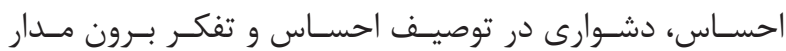

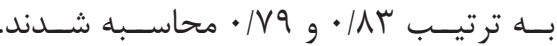
تجزيه و تحليل دادهها

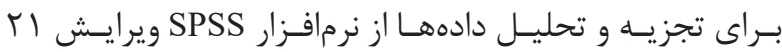

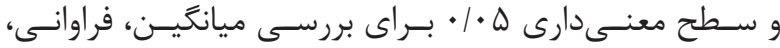

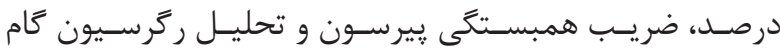

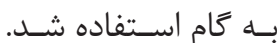

نافته ها

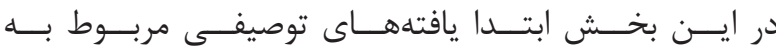

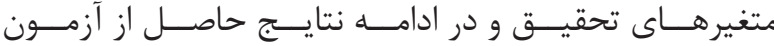

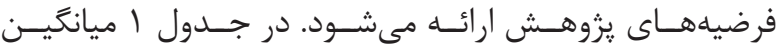

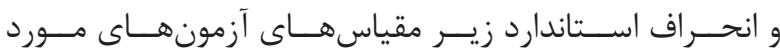

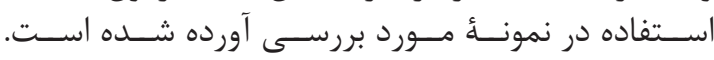

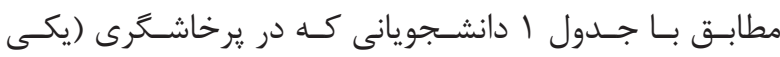

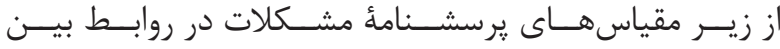

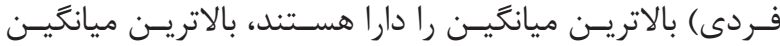

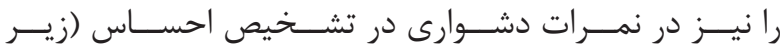

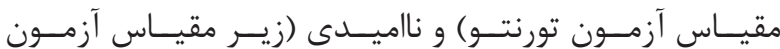

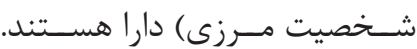

جدول ا- ميانكَين و انحراف استاندارد زير مقياسهاى آزمونهاى تورنتو، آزمون شخصيت مرزى و يرسشنامةٔ مشكلات بين فردى.

\begin{tabular}{|c|c|c|c|c|c|}
\hline كمينه & بيشينه & انحراف استاندارد & ميانگين & زير مقياسها & مقياس \\
\hline re & $\Lambda$. & $\Lambda / 9$ & $\Delta V / F$ & دشوارى در تشخيص احساس & \multirow{3}{*}{ آزمون تورنتو } \\
\hline rq & VI & $\Delta /+1$ & $p q / q$ & دشوارى در توصيف احساس & \\
\hline rF & $\Delta \Delta$ & $\Delta / r^{\prime}$ & $f 1 / d$ & تفكر برون مدار & \\
\hline 19 & f. & $f / d$ & rr/G & ناميدى & \multirow{3}{*}{ شخصيت مرزى آزمون اختلال } \\
\hline f & 19 & $r / 9$ & $I T / T F$ & تكانش ترىى & \\
\hline$v$ & rf & $r / r$ & $I V / F$ & علاينم تجزيهالى و يارانوئيدى & \\
\hline 11 & re & $p / q$ & $r 9 / 9$ & صراحت و مردم آميزى & \multirow{6}{*}{ فركلات بين } \\
\hline f & 10 & $1 / 97$ & $15 / 99$ & كشودكَى & \\
\hline $18 / 9$. & $19 / 9$. & $|/ F|$ & IV/rr & در نظر ترفتن ديكَر ان & \\
\hline 119 & fr & $11 / \mathrm{V}$ & $\Lambda 9 / \%$. & لمبرخاشكرى & \\
\hline If & rq & $p / q$ & $r V / I I$ & حمايتثرى & \\
\hline$r$ & 10 & $F / r 1$ & ir & مشاركت و وابستكّى & \\
\hline
\end{tabular}


جدول r- درصد همزمانى بين نمرات ويزگگ هاى ناكويى هيجانى و شخصيت مرزى بهترتيب در دختران دانشجو با مشكلات در روابط بين فردى.

\begin{tabular}{|c|c|c|c|}
\hline كل & مشكلاتد دميوشى در بروزي & مشكاجلات در روابط بين فرشى دروزى & \\
\hline १४ & $\Gamma \Lambda(\% / \mathrm{r \Delta / \Gamma})$ & $\Delta 9(\%$ \% / & ويزّكىهاى آلكسى تايميا \\
\hline Qr & $r+(\% / r)$ & Tr(\%) & اختلال شخصيت مرزى \\
\hline 10 . & $\Delta \Lambda(\% / \Gamma \Lambda / 9)$ & $9 r(\% / 91 / r)$ & 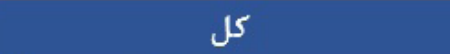 \\
\hline \multicolumn{4}{|c|}{ درصد نوع علايم ناتويى هيجانى و شخصيت مرزى در دختران دانشجو با مشكلات در روابط بين فردى } \\
\hline & اختلال شخصيت مرزى & نائويى هيجانى & علايم \\
\hline & $\% \Delta \varphi$ & $\% 99 / \Delta$ & دشوارى در تشخيص احساس \\
\hline & $\% \Delta \Delta$ & $\% 91$ & دشوارى در توصيف احساس \\
\hline & $\% \pi r / V$ & $\%$ & تفكر برون مدار \\
\hline & $\% 9 r / 1$ & $\% \Delta r$ & ثااميلى \\
\hline & $\% \Delta r / \Lambda$ & $\% 19$ & تكانش ترى \\
\hline & $\%$ & $\%$ & علايه تجزيهاى و يار انوئيدى وايسته \\
\hline
\end{tabular}

جدول بـ ماتريس همبستگى بين مقياسها و مؤلفههاى آن در دختران دانشجو.

\begin{tabular}{|c|c|c|c|c|c|c|c|c|c|c|c|c|}
\hline ir & 11 & $1 \cdot$ & 9 & $\wedge$ & $v$ & 9 & $\Delta$ & $f$ & $r$ & r & 1 & \\
\hline & & & & & & & & & & & 1 & دشوارى در تشخيص احساس \\
\hline & & & & & & & & & & 1 &.$/ 49$ 䄅䄅 & دشوارى در توصيف احساس \\
\hline & & & & & & & & & 1 & $\cdot / r V$ 䄅漛 & 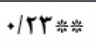 & تفكر برون مدار \\
\hline & & & & & & & & 1 & $+/ T F=$ & $+/ \gamma q \div$ & - $/ \Delta T+\frac{\mathrm{*}}{*}$ & ناميلى \\
\hline & & & & & & & 1 & .199 䄅落 & $+/ T V=$ & $\cdot / T \Lambda$ 粦 & •/rV紫签 & تكانش تُرى \\
\hline & & & & & & 1 & $+\mid \Delta \Delta$ 拳卷 & $+/ \vee \wedge$ 券帣 & $+/ T \Lambda \div$ & $\cdot / \pi r \%$ & $+/ T \Lambda$ 拳卷 & علايم تجزيهاى و يارانوئيسى \\
\hline & & & & & 1 & 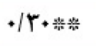 & •/Tr䅈巻 & $+/ 19 \div$ & $+/ 19 *$ & $+/ 10$ & $+/ / F=$ & صراحت و مردم آميزى \\
\hline & & & & 1 & 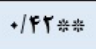 &.$/ 19 \%$ & $.|\pi| \div$ & $+/ 1 \mathrm{~V}$ & $\cdot / T F=$ & $+/ T+*$ & $. / T T \div$ & حمايت ترى \\
\hline & & & 1 & $+/ / F$ &.$/ T V \%$ &.$/ 19 \%$ & $\cdot / 1 \mathrm{~V}$ & $+1+1$ & $+/ 1$ & $\cdot / r$ & $+1+1$ & تحشودَّى \\
\hline & & 1 & $\cdot / T \Delta \div$ & $+/ Y V \frac{*}{2}$ & - / r 䄅漛 & $-+/ T T \div$ & $+/ / F=$ & - $/ Y T$ 䄅券 & $+/ T+*$ & $+/ T F_{\frac{*}{2}}$ & $+/ T T=$ & در نظر كرفتن ديكَران \\
\hline & 1 &.$/ 1 r$ & .1 .9 & . $/ 1 T$ & $\cdot / T V$ 券 & $+/ Y F_{\text {䄅前 }}$ & - / Y 9 拳益 & $\cdot / T \Delta \div$ & $+1 \cdot \Delta$ & $\cdot|\pi|=$ & - $/ T Y$ 崇漛 & يرخاشعَرى \\
\hline 1 & $* 1+\Delta$ & $+/ T \Delta$ & $\cdot / T V=$ & $+/ Y \Lambda$ 䄅帣 & $+/ Y F_{\text {券䖭 }}$ & $\cdot / T T=$ & $+/ T r^{*}$ & $+/ T Y$ 拳券 & */IT &.$/ 19$ & $+/ / F=$ & مشاركت و همبستَّى \\
\hline
\end{tabular}

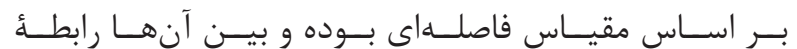

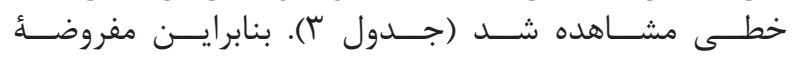

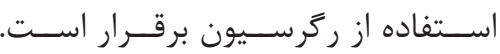

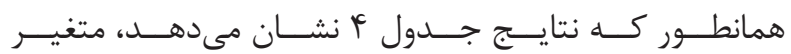

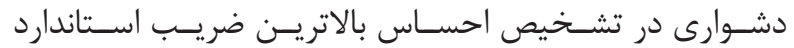

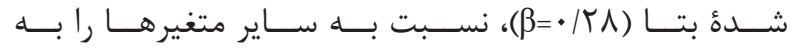

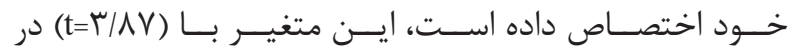

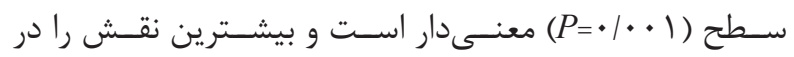

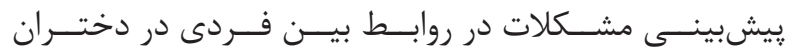

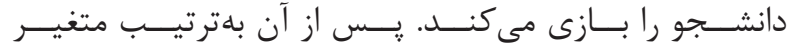

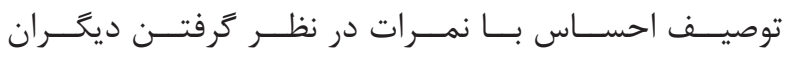

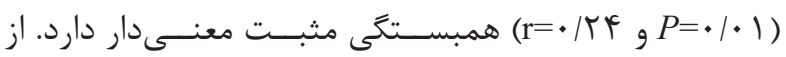

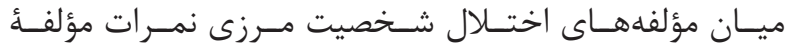

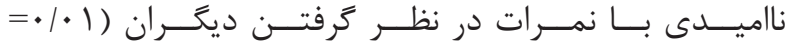

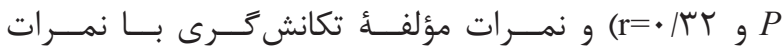

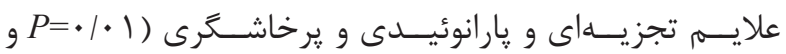

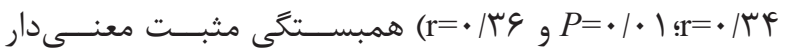

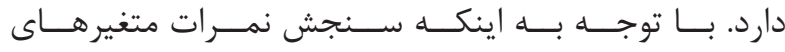

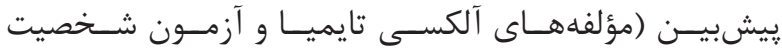

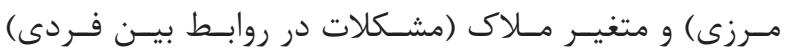


جدول F- ضرايب حاصل از كام ينجم تحليل ركرسيون كام به كام مؤلفهاى ناكويى هيجانى، اختلال شخصيت مرزى بر مشكلات در روابط بين فردى.

\begin{tabular}{|c|c|c|c|c|c|}
\hline معنى دارى & $\mathbf{T}$ & $\boldsymbol{\beta}$ & خطاى معيار & B & 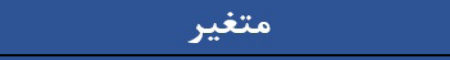 \\
\hline$\cdot 1 \cdot \bullet \Delta$ & $r / \wedge q$ & & 1.119 & $r q / 41$ & عدد ثابت \\
\hline$\cdot 1+\cdot 1$ & r/Av & $\cdot / \mathrm{r} \Lambda$ & $r / T r$ & N/gF & دشوارى در تشخيص احساس \\
\hline$\cdot 1 \cdot \cdot v$ & $r / V \Delta$ & $\cdot / 4$ & . &.$|8|$ & دشوارى در توصيف احساس \\
\hline$\cdot 1+11$ & $r / \Delta V$ &.$/ 11$ & 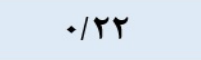 & $\cdot 1 \Delta 9$ & ناميدى \\
\hline$\cdot 1 \cdot+r$ & $r /+1$ &.$/ 4 t$ &.$/ 1 V$ & .10 & تكانش \\
\hline$\bullet 1 * 1$ & $r / 9 V$ & $\cdot / T$ & .119 & - IFF & علايم تجزيهاى و هارانوئيدى \\
\hline
\end{tabular}

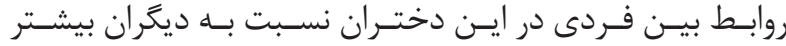

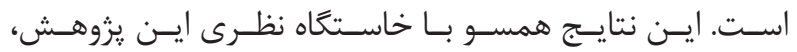

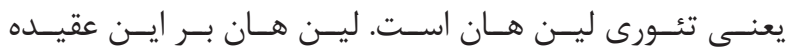

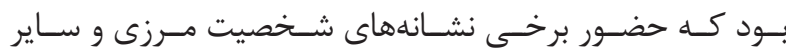

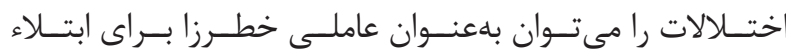

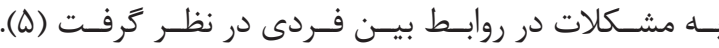

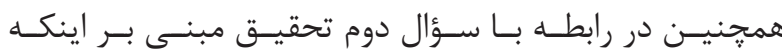

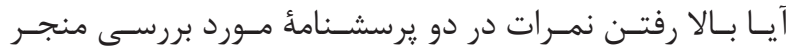

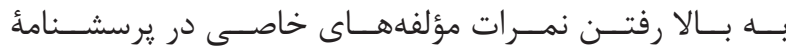

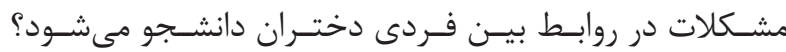

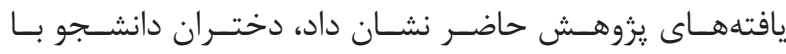

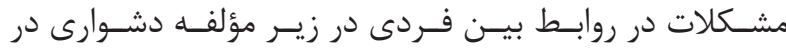

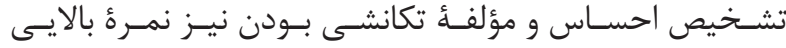

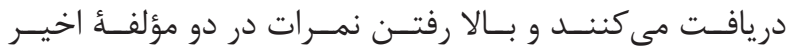

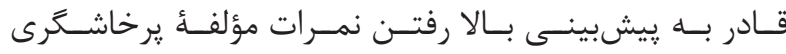

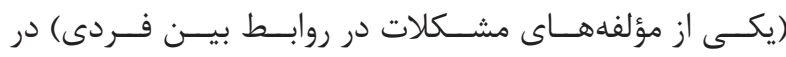

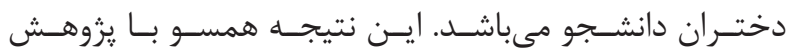

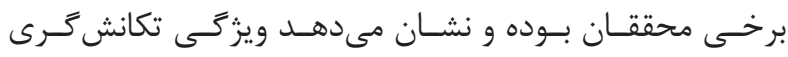

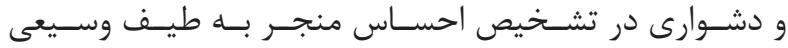

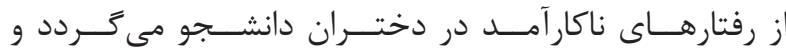

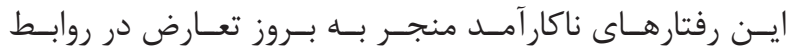

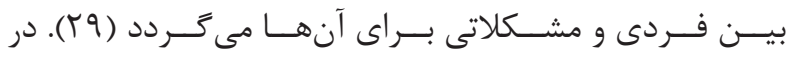

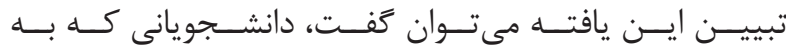

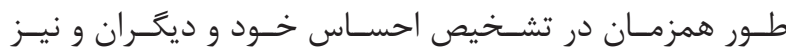

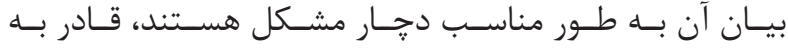

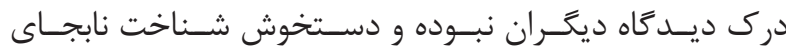

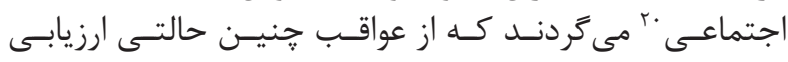

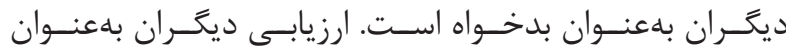

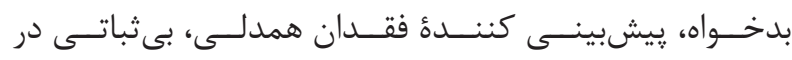

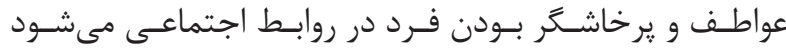

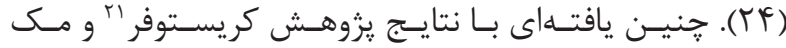

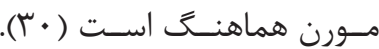

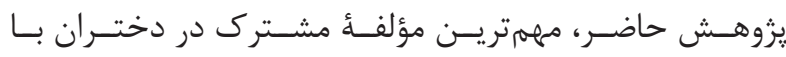

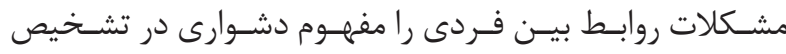

${ }^{19}$ Webb and McMurran

${ }^{20}$ Aberrant social cognition

${ }^{21}$ Christopher

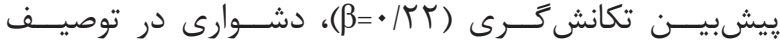

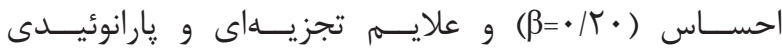

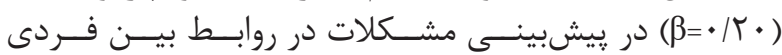

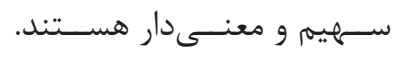

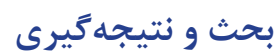

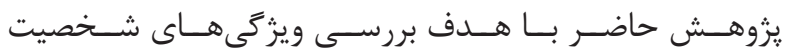

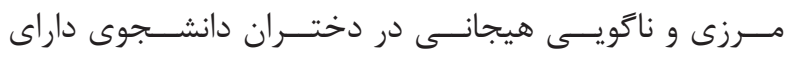

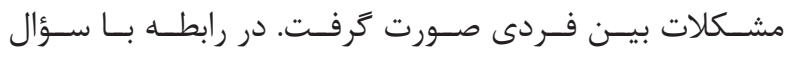

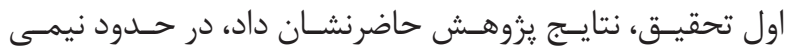

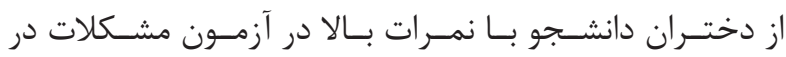

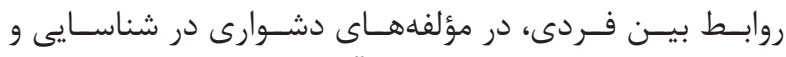

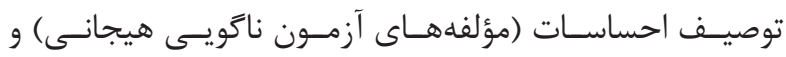

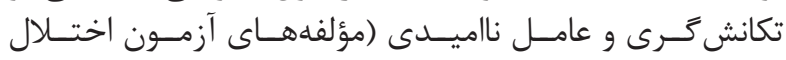

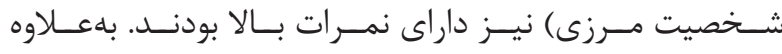

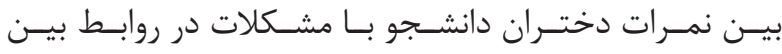

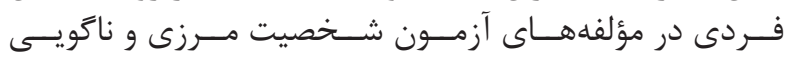

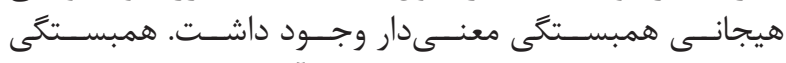

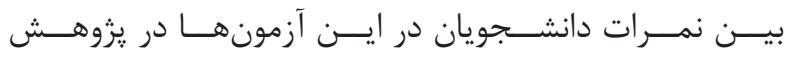

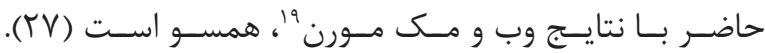

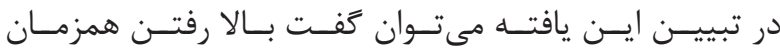

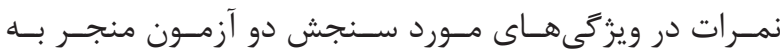

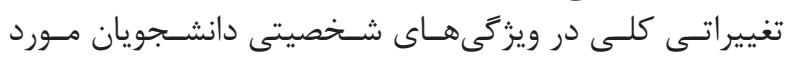

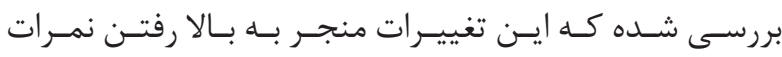

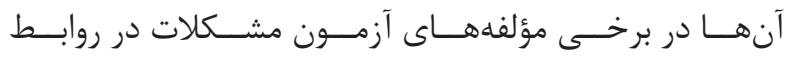

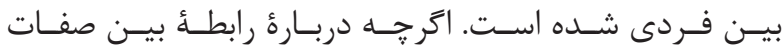

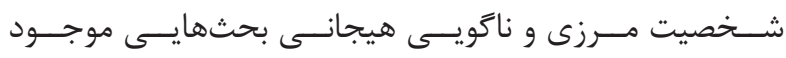

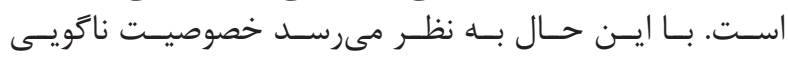

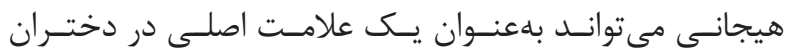

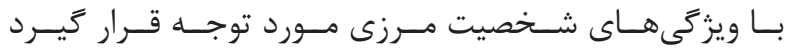

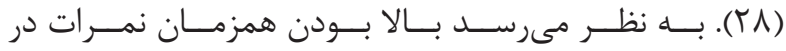

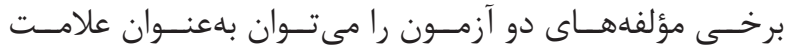

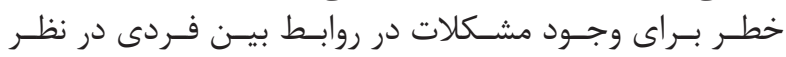

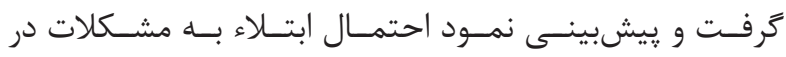




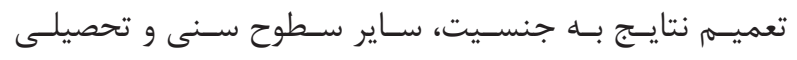

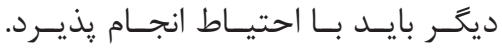

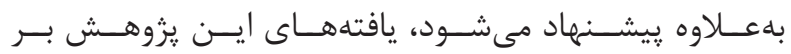

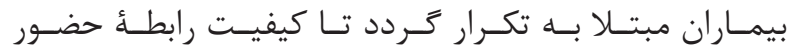

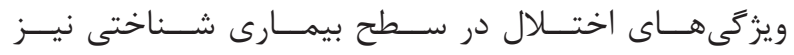

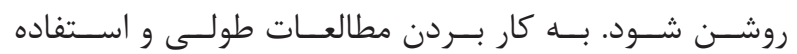

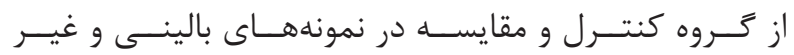

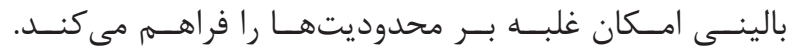

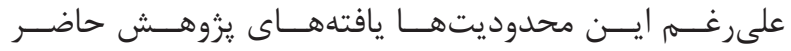

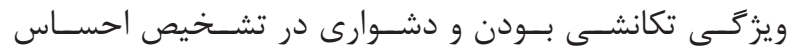

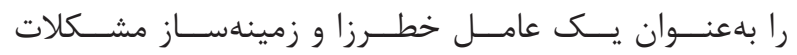

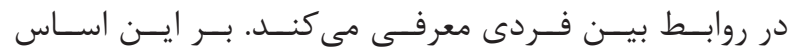

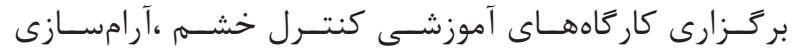

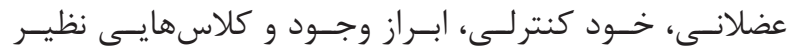

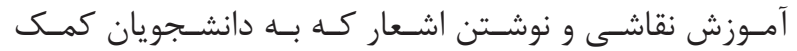

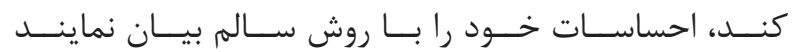

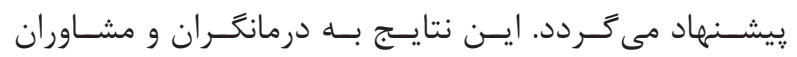

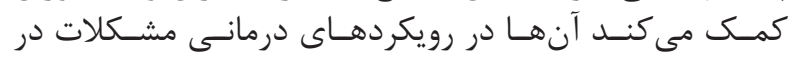

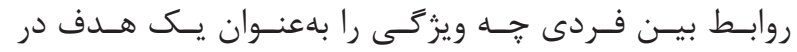

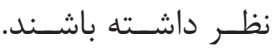

1.Vanheule S, Desmet M, Rosseel Y, Verhaeghe P, Meganck R. Relationship patterns in alexithymia: a study using the core conflictual relationship theme method. Psychopathology. 2006; 40: 14-21.

2.Vanheule S, Desmet M, Meganck R, Bogaerts S. Alexithymia and interpersonal problems. J Clin Psychol. 2007; 63(1): 109-17.

3. Chabrol H, Rousseau AL, Callahan S, Hyler SE. Frequency and structure of DSM-V personality disorder traits in college students. Pers Individ Dif. 2007; 43: 1767-76.

4. Bischof A, Meyer C, Bischof G, Kastirke N, John U, Rumpf HJ. Comorbid axis I-disorders among subjects with pathological, problem, or at-risk gambling recruited from the general population in Germany: results of the PAGE study. Psychiatry Research. 2013; 210(3): 1065-70.

5. Linehan MM. Cognitive-behavioral treatment of borderline personality disorder. New York: Guildford Press. 1993; p. 260-4.

6. Vihang NV. Diagnostic and statistical manual of mental disorders. 5. A quick glance. Indian J Psychiatry. 2013; 55(3): 220-3.

7. Loas G, Speranza M, Pham-Scottez A, Perez-Diaz F, Corcos M. Alexithymia in adolescents with borderline

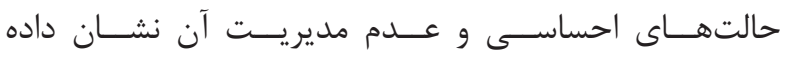

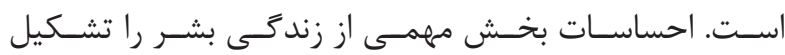

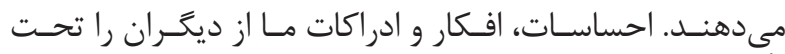

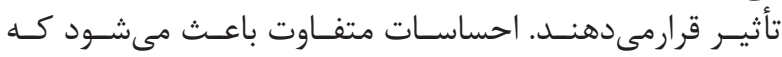

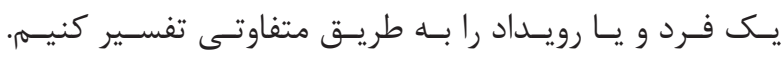

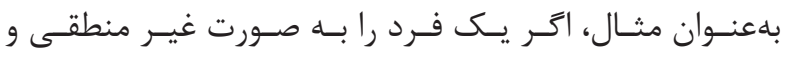

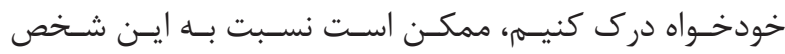

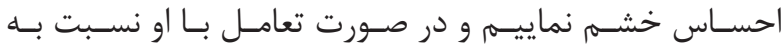

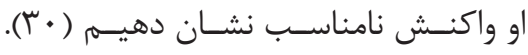

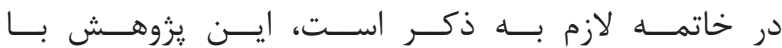

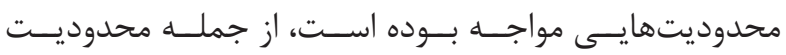

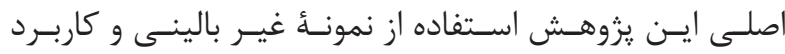

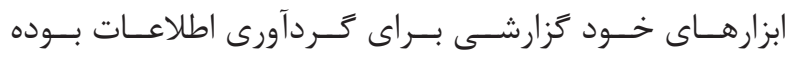

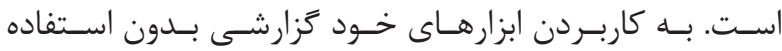

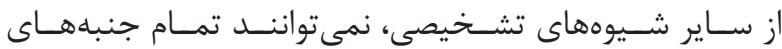

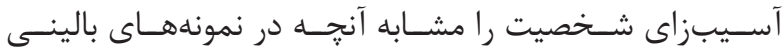

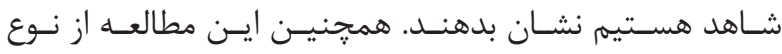

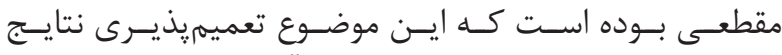

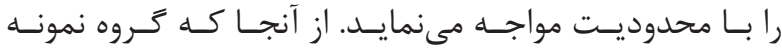

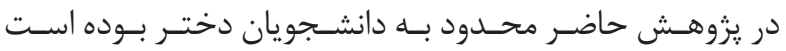

منابع

personality disorder. J Psychosom. 2012; 72(2): 147-52.

8. Sharp C, Pane H, Ha C, Venta A, Patel AB, Sturek J, et al. Theory of mind and emotion regulation difficulties in adolescents with borderline traits. J Am Acad Child Adolescent. Psychiatry. 2011; 50: 563-73.

9. Bornovalova MA, Lejuez CW, Dauhters BB, Rosenthal MZ, Lynch TR. Impulsivity as a common process across borderline personality and substance use disorders. Clin Psychol Rev. 2005; 25: 790-812.

10. BachM de, Zwaan M, Ackard D, Nutzingzer DO, Mitchell JE. Alexithymia: relationship to personality disorders. Compr Psychiatry. 1994; 35: 239-43.

11. Taylor GJ, Bagby RM, Parker JD. A. The 20-Item Toronto Alexithymia Scale. IV. Reliability and factorial validity in different languages and cultures. J Psychosom Res. 2003; 55(3): 277-83.

12. Guttman H, Laporte L. Alexithymia, empathy, and psychological symptoms in a family context. Compr Psychiatry. 2002; 43(6): 448-55.

13. Berenbaum H. Childhood abuse, alexithymia and personality disorder. J Psychosom Res. 1996; 41 (6): 585-95.

14. Eastwood JD, Cavaliere C, Fahlman SA, Eastwood AE. A desire for desires: Boredom and its relation to 
alexithymia. Pers Individ Dif. 2007; 42: 1035-45.

15. Skodol AE, Gunderson JG, McGlashan TH, Dyck IR, Stout RL, Bender DS. Functional impairment in patients with schizotypal, borderline, avoidant, or obsessivecompulsive personality disorder. Am J Psychiatry. 2002; 159(2): 276-83.

16. Wachs K, Cordova JV. Mindful relating: exploring mindfulness and emotion repertoires in intimate relationships. J Marital Fam Ther. 2007; 33(4): 464-81.

17. Fonagy P, Luyten P. A developmental, metallizationbased approach to the under Standing and treatment of borderline personality disorder. Dev Psychopathol. 2009; 21(4): 1355-81.

18. Modestin J, Furrer R, Malti T. Study on alexithymia in adult non-patients. J Psychosom Res. 2004; 56(6): 707-9.

19.Yousefi F. The relationship between emotional intelligence and communication skills in university students. Quarterly Journal of Iranian Psychologists. 2006; 3(9): 5-13.

20. Heidarpour S, Dokane Fard F, Bahari S. The impact of effective communication skills training to increase self-esteem and reduce their shyness physical-motor in Tehran. Quarterly Journal of New Ideas in of Educational Sciences. 2008; 3: 41-51.

21. Jahani Hashemi H, Rahimzadeh Mirmahalleh S, Ghafelehbashy H, Sarichloo ME. Mental health of Qazvin university students in 2005. The Journal of Qazvin University of Medical Sciences. 2008; 12(2): 41-9.

22. Eagly AH, Johannesen-Schmidt MC, Van Engen ML. Transformational, transactional and laissez-fair leadership styles: a meta-analysis comparing men and women. Psychol Bull. 2003; 129(4): 569-91.
23. Barkham M, Hardy GE, Startup M. The IIP 32: a short version of the inventory of interpersonal problems. Br J Clin Psychol. 1996; 35(1): 21-35.

24. Fath N, Azad Fallah P, Tabatabaei SK. Rahimi Ch. The validity of interpersonal problems questionnaire. J Clin Psychol. 2013; 5(3): 69-80.

25. Mohammad Zadeh A, Goodarzi A, Taghavi M, Molazadeh JF. Actor structure, validity, reliability, and standardization of borderline personality scale in shiraz university students. Journal of Fundamentals of Mental Health. 2005; 7(27): 75-89.

26. Bagby P, Taylor I. The twenty-item toronto alexision IV. maastricht: department of thymia scale-i. item selection and. medical, clinical and experimental crossvalidation of the factor psychology, maastricht university, ture. Journal of Psychosomatic Re Netherlands. 1994; 38: 23-32.

27. Besharat MA. Psychometric characteristics of Persian version of the Toronto Alexithymia scale-20inclinical and nonclinical samples. Iranian Journal of Medical Science. 2008; 33(1): 1-6.

28. Webb DJ, McMurran M. Emotional intelligence, alexithymia, and borderline personality disorder traits in young adults. J Pers Soc Psychol. 2008; 2(4): 265-73.

29.Donegan NH, Sanislow CA, Skudlarski P. The structural inter hyperactivity in borderline personal ity disorder: Implications for emotional personality disorders: psychotherapeudysregulation. New Haven: Yale University. 2003; 54: 1284-93.

30. Christopher G, McMurran M. Alexithymia, empathic concern, goal management, and social problem solving in adult male prisoners. Psychology, Crime, and Law. 2009; 15(8): 697-709. 